UDK 811.112.2'373.7

DOI https://doi.org/10.32841/2409-1154.2019.40.2.12

\author{
Lalaian N. S., \\ $P h D$, \\ Dozentin am Lehrstuhl für germanische Philologie und Translation \\ Nationale Taras-Schewtschenko-Universität Kyiv
}

\title{
PHRASEOLOGISMEN UND TEXT: PROBLEME DER PHRASEOLOGISCHEN MODIFIKATION
}

\begin{abstract}
Лалаян Н. С. Фразеологізми і текст: проблеми фразеологічних модифікацій
\end{abstract}

Анотація. Статтю присвячено дослідженню функціонування фразеологізмів сучасної німецької мови у художніх текстах. Визначено основні відмінності фразеологізмів від вільних словосполучень (лексико-семантичну цілісність, ідіоматичність, лексикалізацію, відтворюваність); проаналізовано особливості функціонування фразеологізмів у художніх текстах; розглянуто питання текстотвірних потенцій фразеологічних одиниць і виокремлено найважливіші характеристики фразеологізмів, які визначають їхні текстотвірні потенції; висвітлено відмінності між фразеологічними варіантами, варіаціями та фразеологічними модифікаціями, 3'ясовано підходи до трактування цих понять різними мовознавцями; на прикладі художніх текстів проаналізовано основні типи модифікацій фразеологізмів. Доведено, що мовленнєві перетворення не $\epsilon$ випадковими чи помилковими фактами, а типовим способом вживання фразеологізмів, який викликаний певною комунікативно-прагматичною метою і належить до зумовлених системою властивостей фразеологізмів. Основними причинами утворення та використання фразеологічних модифікацій $є$ : вплив на реципієнта, підсилення експресивності й образності висловлювання, ущільнення інформації, досягнення когерентності тексту, отримання певного каламбурного ефекту, номінація. На основі прикладів iз художніх творів німецьких авторів проаналізовано явища фразеологічної субституції (лексичної, прагматично зумовленої або зумовленої текстом заміни компонентів звороту), експансії (розширення компонентного складу фразеологізму), редукції (скорочення компонентного складу фразеологізму), контамінації (злиття в одному виразі двох чи кількох фразеологізмів) та їхні комбінації.

Ключові слова: фразеологізм, функціонування, художній текст, текстотвірні потенції, модифікація, субституція, експансія, редукція, контамінація.

Untersuchungsgegenstand und Problemstellung. Im Zusammenhang mit der Entfaltung der Textlinguistik und der Untersuchung kommunikativ-pragmatischer Aspekte der Sprache wird heute auch die Erforschung der Verwendung der Phraseologismen in der gesellschaftlichen Kommunikation, werden ihre spezifischen Möglichkeiten, zum Aufbau der Textstruktur beizutragen, als Schwerpunkt der Phraseologieforschung betrachtet [1, S. 216; 2, S. 41].

Im Mittelpunkt der folgenden Abhandlung stehen die Verwendungsbesonderheiten der phraseologischen Einheiten (Phraseologismen, Phraseme) und zwar die okkasionellen Modifikationen usueller Phraseologismen. Die Notwendigkeit, weitere Untersuchungen auf diesem Gebietdurchzuführen, besteht darin, dass ,die semantischen Prozesse, die sich mit Phraseologismen im Kontext abspielen können " nicht ausreichend erforscht sind [3, S. 321]. Darüber hinaus wird heutzutage auf die kommunikative und didaktische Funktion der Texte, deren Inhalt und deren Gestaltung große Aufmerksamkeit der Linguisten gerichtet. Da gerade Phraseologismen Sprachmittel sind, die den für den Text im Fremdsprachenunterricht geforderten Rezeptionsanreiz mitschaffen [3, S. 321], sollten sie gründlicher unter die Lupe genommen werden. Bei der Analyse richtet sich unser Augenmerk auf das Funktionieren der Phraseologismen vor allem in Belletristik. In der folgenden Abhandlung gehen wir auf solche Fragen ein: Kriterien für die Differenzeirung der Phraseologismen von den freien Wortverbindungen; Besonderheiten der Verwendung von Phraseologismen in den Texten der schönen Literatur; textbildende Potenzen von Phraseologismen; phraseologische Varianten und phraseologische Modifikationen; Arten der phraseologischen Modifikationen.

Hauptgrundlagen des Artikels. Phraseologismen sind „feste“ (fixierte) syntaktische Konstruktionen mit den Merkmalen der lexikalisch-semantischen Stabilität, Idiomatizität, Lexikalisierung und Reproduzierbarkeit [1, S. 35]. Die lexikalisch-semantische Stabilität oder Festigkeit besteht darin, dass der Austausch der phraseologischen Komponenten innerhalb der Wendung im Vergleich zu freien syntaktischen Verbindungen begrenzt und in vielen Fällen gar nicht möglich ist. Die lexikalisch-semantische Stabilität der Phraseologismen zeigt sich auchin landschaftlich-territorialen Dubletten. So wird Rahm als einzelnes Lexem vorwiegend westmitteldeutsch, süddeutsch, österreichisch und schweizerisch gebraucht, während im übrigen Sprachgebiet dafür Sahne üblich ist. Aber in den Phraseologismen den Rahm abschöpfen - ,sich das Beste nehmen“, den Rahm ansetzen lassen - ,abwarten“ ist solcher Ersatz unüblich. Zur Stabilität der Phraseologismen tragen außerdem phraseologisch gebundene Wörter oder „unikale Komponenten“, die im freien Sprachgebrauch entweder als veraltet gelten, oder an eng fachsprachlichen Gebrauch gebunden sind (z.B. jemandem den Laufpass geben - „sich von jemandem trennen, jemanden wegschicken“, auf Anhieb - ,sofort"). Syntaktische und morphologisch-flexivische Anomaliensind ein weiteres Merkmal der Stabilität der phraseologischen Einheiten, die sich einerseits in der syntaktischen Verbindung der Komponenten eines Phraseologismus, andererseits in Beschränkungen der Transformationen, der transformationellen Defektivität zeigen (z.B. gut Ding hat schweren Anfang - ,es ist schwer etwas anzufangen“, Hals über Kopf - ,in höchster Eile“), Stabilität nichtidiomatischer Komponenten (z.B. Wortpaare wie Freund und Leid, hier und da, hin und her ) [1, S. 37-64]. Die Idiomatizität der Phraseologismen entsteht auf dem Wege der Metaphorisierung (z.B. jemanden auf die Palme bringen - ,jemanden wütend machen“, in der Tinte sitzen - ,,in einer unangenehmen Lage sein") sowie durch die Spezialisierung der nichtübertragener Sememe in bestimmten Verbindungen (z.B. sich auf 
den Weg machen - „zu einem bestimmten Zweck fortgehen“, alle, die es angeht - ,alle Interessenten“).Unter Lexikalisierung der Phraseologismen versteht man ihre Speicherung im Lexikon, was bedeutet, dass sie nicht mehr nach einem syntaktischen Strukturmodell in der Äußerung ,produziert“, sondern dass sie als „,fertige“ "lexikalische Einheitenin der Rede „reproduziert“ werden [1, S. 67].

Das Verhältnis von Phraseologie zu Literatur ist gekennzeichnet durch den kreativen Gebrauch von Phrasemenin literarischen Texten. Die Verwendung von Phraseologismen in Texten der schönen Literatur trägt der ästhetisch-künstlerischen Struktur des Werkes bei. Sie dienen in erster Linie der Personencharakteristik durch Sprachporträt, kreativer Auflösung des „Schemas der Benennung“, „,kontrastierendem In-Frage-Stellen“ [1, S. 230], außerdem wird der Phraseologismus als „Hauptmittel der Vertextung“ eingesetzt und „bestimmt als solches nicht nur die Struktur des Textes, sondern auch die Art und Weise der künstlerischen Gestaltung sowie den konnotativen Gehalt des Textes“" [5, S. 692]. Die Phraseologismen dienen „der Schließung einer Benennungslücke“ und gelten als „expressive Konkurrenzformen“ zu bereits vorhandenen nichtexpressiven Benennungen [1, S. 167]. Sie ermöglichen Textkohärenz sowie sprachspielerische Betätigung [6, S. 239]. Die verschiedenartigen Leistungen ergeben sich aus den oben erwähnten strukturell-semantischen Eigenschaften der Phraseologismen sowie aus ihrer Ambiguierung durch den Text (betrifft vor allem Phraseologismen, die infolge der Metaphorisierung entstanden sind und besteht darin, dass „,mit der phraseologischen Gesamtbedeutung gleichzeitig eine konstruktionsexterne Bedeutung einzelner Komponenten oder auch eine nichtphraseologische Bedeutung der ganzen Konstruktion aktualisiert wird" [7, S. 142].

Die Etabliertheit der Phraseologismen macht sie zum Bestandteil des Phraseolexikons einer Sprache, zum sprachlichen Zeichen mit Formativ und Bedeutung. Sie haben mit diesem Status jedoch nicht ihren dynamischen Charakter verloren, sondern dieser wird aufgelockert durch Variationen, Modifikationen, polysemische Bedeutungsdiffusionen und im Text durch Kontexteinflüsse [4, S. 111].

Das Auftreten von Phraseologismen in Texten ermöglichen ihre textbildenden Potenzen. Der Begriff, ,textbildende Potenzen der Phraseologismen“ wurde von I. Čhernyševa eingeführt. Weiterentwickelt wurde das Konzept insbesondere von D. Dobrovol'skij, W. Fleischer, B. Wotjak, A. Sabban. B. Wotjak hebt mit Recht hervor, dass Phraseologismen als systemhaft relativ stabile, polylexikale Einheitendurch, ,eine hohe, in der Rede aktualisierbare textbildende (kreative Verknüpfungs-, Assoziations- und Modifikations-) Potenz, ein sehr reiches kommunikatives Potential "gekennzeichnet sind. Dieses Potenzial ergibt sich daraus, dass es sich bei den Phraseologismen zwar um feste formal-semantische ganzheitliche Einheiten mehrgliedrigen Charakters handelt, die aber für den jeweiligen Kontext und je nach kommunikativer Situation ihre Merkmale aufheben können und vielfältige Möglichkeiten der textuellen Aktualisierung bieten [8, S. 100].

$\mathrm{Zu}$ den wichtigsten Eigenschaften der Phraseologismen, die ihre textbildenden Potenzen bestimmen, zählt W. Fleischer folgende:

1) syntaktische Struktur der Wortgruppe und daraus sich ergebende potentielle Teilbarkeit, syntaktisch-strukturelle Variabilität;

2) semantische Teilbarkeit mit Variationen bis zur semantischen Autonomisierung von Komponenten, zur Derivation neuer Einheiten;
3) „diffuser Charakter“ der Bedeutung eines wesentlichen Teiles der Phraseolexeme;

4) reich entwickelte Synonymik innerhalb der Phraseolexeme;

5) stark entwickelte Expressivität durch Bildlichkeit und Konnotationen; Möglichkeiten der Expressivitätssteigerung [1, S. 216].

Im Prozess der Textproduktion werden sprachliche Einheiten in ihrer Form und Bedeutung auffällig ,aktiviert“ und „aktualisiert“, denn „Textebieten Freiraum zur Entfaltung des phraseologischen Potenzials" [9, S. 103]. Interessant vom Standpunkt der Textgestaltung sind nicht die ,Aktualisierungen usueller Art“ (es geht um usuelle Varianten, die durch grammatische Anpassung im Text entstehen), sondern verschiedenartige okkasionelle Abwandlungen oder „phraseologische Bildungen okkasionell-textgebundener auffälliger Art" [9, S. 103], die man als Modifikationen / Transformationen des phraseologischen Modells betrachtet. Die Ursachen von Modifikationen sind verschieden: sie erwecken beiden Rezipienten bestimmte Reaktionen und rufen Assoziationen hervor; sie tragen der Expressivität des Testes bei; gelten als neue Benennungen, die ein Denotat möglicherweise anschaulicher oder differenzierter benennen als konventionelle, oder als ,situative Füllung der Leerstellen“ [6, S. 239]. Darüber hinaus ist eine nicht unwichtige Ursache die Freude der Textproduzenten und -rezipienten am unterhaltsamen Sprachspiel, am sprachlichen Experiment, das für den Rezipienten vornehmlich als Leseanreiz wirkt und sein Interesse für den Textinhalt befördert [3, S. 322]. Die Modifikationen sind nicht an die usuelle Variabilität gebunden. Die Forscher (B. Wotjak, D. Dobrovol'skij, W. Fleischer) sind sich darüber einig, dass es bei den individuell-okkasionellen Modifikationen nicht um abweichenden oder fehlerhaften Sprachgebrauch geht, dass sie nicht als „Ausnahme“ oder „Sonderfall“ anzusehen sind, sondern dass Modifikationen im Gegenteil eine ganz typische Verwendungsweise der Phraseologismen sind.

Die Abgrenzung zwischen Modifikationen, Variationen und Varianten ist nicht ohne Probleme. So heben H. Burger und B. Wotjakvon den okkasionellen Modifikationen usuelle, konventionelle, nebeneinander existierende Varianten ab [10; 8]. D. Dobrovol'skij und I. Čhernyševa unterscheiden in diesem Zusammenhang zwischen „strukturellen Varianten“ (seine Hand / Hände im Spiel haben; kein Haar / kein Härchen krümmen); „struktureller Synonymie / Antonymie“ (j-n zum besten haben / halten; das Feld behaupten / räumen); ,phraseologischen Serien“ (ein lockerer / lustiger / komischer / seltsamer Vogel; wieder Todvon Basel / Warschau / Ypern aussehen) und „Konversiven“, worunter insbesondere (Aktionsart-)Variationen eines wendungsinternen „neutralen“ Verbs verstanden werden (unter Dach uns Fach sein / bringen / kommen) [8, S. 5]. A. Sabban spricht von Modifikationen im engeren Sinne (es geht dabei um formal veränderte Phraseme), die sie auch als Variationen bezeichnet und von usuellen und grundsätzlich lexikographisch erfassten Varianten abgrenzt [11, S. 245].

W. Fleischer, G. Helbig, G. Lerchner differenzieren in der „Kleinen Enzyklopädie - deutsche Sprache“ die Begriffe der Variation und der Modifikation. Unter Variation verstehen sie das Auftreten von usuellen (konventionellen), lexikographisch zu kodifizierenden und als normgerecht zu beurteilenden morphosyntaktischen oder lexikalischen Alternanten (Varianten) eines Phraseologismus [7, S. 135]. Die Modifikation ist nicht an die usuelle Variabilität gebunden [7, S. 135].

In der „Phraseologie der deutschen Gegenwartssprache“verwendet W. Fleischer Termini ,phraseologische Variationen“, „variierte Phraseologismen", „Strukturvariationen" und „phraseologische 
(Struktur-) Varianten [1, S. 209-210] und unterscheidet drei Typen von Variationen, wobei unter diesem Oberbegriff unterschiedliche Ebenen (Ebene des Systems und Ebene der Verwendung, d.h. ,phraseologische Strukturvarianten“ und „variierte Phraseologismen“ zusammengefasst werden, nämlich: 1) morphologische und teilweise auch syntaktische Veränderungen einzelner Komponenten / „Strukturvariationen" oder „phraseologische Strukturvarianten“, die die innere Organisation des Materialbestandes nicht antasten (z.B. seine Hand / Hände im Spiel haben, mit den Achseln / die Achseln zucken, j-m kein Haar / Härchen krümmen, j-m keinen / nicht den Bissen Brot gönnen) (H. Burger bezeichnet nur diese Gruppe, in der es um Substituierbarkeit von strukturellen Elementen eines Phraseologismus geht, als Varianten im striktem Sinne; bei D. Dobrovol'skij sind das strukturelle Varianten; 2) Austausch einzelner lexikalischer Komponenten des Phraseologismus, wobei in der Regel entweder phraseologische Synonyme (z.B. j-n auf den Arm / auf die Schippe nehmen) oder phraseologische Antonyme entstehen (z.B. mit dem/gegen den Strom schwimmen) (H. Burger betrachtet solche Einheiten nicht als Varianten ein und derselben Nennform, sondern postuliert dafür, dass eine Nennform im onomasiologischen Teil der anderen als Synonym erscheinen sollte; bei D. Dobrovol'skij sind das strukturelle Synonyme) [8, S. 6]; 3) Erweiterung oder Reduktion des Komponentenbestandes im Text.

Im Anschluss an H. Burger und B. Wotjak grenzen wir usuelle Varianten eines Phraseologismus von seinen kontextuellen Modifikationen $\mathrm{ab}$.

Die Modifikation von Phraseologismen ist ein kennzeichnendes Merkmal ihres Gebrauchs. Nach H. Burger gebe es kaum eine Veränderung eines Phraseologismus, die in irgendeinem Kontext nicht möglich und durchaus sinnvoll wäre [10, S. 28]. Allerdings muss berücksichtigt werden, dass die phraseologische Basis bestimmt, ob und in welcher Weise die Veränderungen auftreten können, so dass keine absolute Freiheit der Wahl der Modifikationsarten und -mittel gegeben ist [3, S. 322]. Der Eingriff in die innere Struktur eines Phraseologismus setzt voraus, dass die phraseologische Basis bis zu der formalen und semantischen Grenze erhalten werden muss, die ihre Identifikation gerade noch erlaubt und die sichert, dass der Rezipient den Text mit der Modifikation auch versteht [3, S. 322]. Der Leser soll die usuelle Form eines Phraseologismus kennen, denn ,nur wenn der Rezipient die phraseologische Basiskennt, kann er bestimmte Abweichungen im Zusammenspiel von paradigmatisch bedingter bzw. textgebundener Modifizierung und Assoziierung einerseits und Systemvorgaben andererseits einordnen, dekodieren, interpretieren und die Freude am Spielteilen“" [8, S. 103]. Wenn eine vom Sender vorausgesetzte bestimmte Kooperationsbereitschaft, bzw. Kooperationsfähigkeit (als Gelingensbedingung) aus welchen Gründen auch immer (z.B. Unkenntnis der phraseologischen Basis, unterschiedliche Wissensvoraussetzungen / fehlendes gemeinsames Wissen über einen Sachverhalt, intellektuelle Überforderung...) beim Rezipienten fehlt, wird das „Vergnügen“ an bewussten Abweichungen von der phraseologischen Ausgangsform nicht geteilt, wird die Wirkungsabsicht des Senders nicht erkannt und eine bestimmte Verwendung möglicherweise als Fehlerbetrachtet [8, S. 104].

Die Modifikationen von Phraseologismen operieren mit Substitution, Erweiterung / Expansion, Reduktion, Kontamination und es treten auch die unterschiedlichsten Kombinationen dieser Modifikationen auf.

Man verwendet phraseologische Modifikationen in großem Maße absichtlich, um eine bestimmte Wirkung zu erzielen. Dabei geht es vor allem um solche pragmatisch-semantischen Gründe wie Erhöhung des Rezeptionsanreizes, Verstärkung von Aussagen, Informationsverdichtung, Erreichung eines bestimmten wortspielerischen Effekts, Anschaulichkeit, Expressivität oder auch Nomination [9, S. 103]. Die okkasionellen Modifikationen markieren den Text in einer ganz besonderen Weise, rufen textspezifische Konnotationen hervor. Darin kommt eine wesentliche textbildende Funktion phraseologischer Wortfügungen zum Ausdruck. Ersichtlich wird es vor allem an phraseologischen Kontaminationen und textgebundenen Substitutionen:

1. Das war ein mannhaftes Wort! Ich hatte befürchtet, er würde herumreden, wie die Katze um den heißen Brei und mit einem halben Versprechen den Rückzug antreten [12, S. 85]. Zugrunde liegt eine Kontamination der Phraseologismen um den heißen Brei herumreden - „um etwas herumreden, nicht über Kern der Sache reden" und wie die Katze um den heißen Brei herumschleichen „nicht wagen, eine schwierige Sache anzupacken; sich nicht an eine heikle Sache wagen“.

2. „Siehst du, mein Guter“, sagte er, „so leben wir. Klein, aber allein" [12, S. 24]. Nach: klein, aber fein.

3. „Gelehrter Barhocker“, lobte Eugen. „Der Apfel fällt nicht weit vom Pferd"[12, S. 35]. Nach: Der Apfel fällt nicht weit vom Stamm.

4. Prima, Dan. Man muss die Mädchen schmieden, solange sie warm sind [12, S. 65]. Nach: Man muss das Eisen schmieden, solange es heiß ist.

Die Verwendung der textbezogenen neuen Komponenten ist für den Leser auffällig, unterhaltsam und anregend. Bei der Dekodierung der komprimierten Information muss er gleichzeitig die Basisbedeutung des Phraseologismus und den Textbezug erfassen.

Bei pragmatisch bedingter Substitution werden die Basiskomponenten mit Synonymen, Antonymen oder Hyperonymen ausgetauscht:

5. Der Duft der Suppe ließ mir das Wasser über den Gaumen laufen [12, S. 82]. Nach: j-m das Wasser im Mund zusammenlaufen lassen / j-m läuft das Wasser im Mund zusammen - ,sich auf die bevorstehende Mahlzeit freuen".

6. Der Schlag würde ihn treffen, wenn er sehen könnte, wie meilenweit wir vom rechten Pfade abgekommen waren [12, S. 233]. Nach: vom rechten Weg(e) / Kurs abkommen -,, kriminell, lasterhaft werden".

Solche Modifikationen sind aber kaum expressiver als ihre Basen und kommen nicht so oft vor.

Die phraseologischen Modifikationen haben vielfältige textkonstitutive und pragmatische Funktionen. Die Substitution und Expansion der Phraseologismen tragen zur Textkohärenz bei. Durch den Ersatz oder die Erweiterung im Komponentenbestand werden in die Wendung neue Elemente eingebracht, die den Phraseologismus an den Kontext semantisch anpassen. Dadurch gewinnt der Text an inhaltlicher Geschlossenheit. Die phraseologische Basis bleibt erhalten und wird zusätzlich um neue kontextrelevante Elemente bereichert, die konkretisierend und spezifizierend wirken [9, S. 104]:

7. Mag er arm sein wie Hiob nach der Währungsreform, mir gefällt er [12, S. 16].

8. Wer wagt, gewinnt oder bekommt Prügel [12, S. 182].

9. Matschats Hahn saß Aehre wie ein spitzer, kalter Dorn im Leib [13, S. 85]. Nach: etwas sitzt j-m wie ein Dorn im Leibe - ,für jemanden sehr ärgerlich / unerträglich sein“.

10. Der Ober machte ein Gesicht, als ahnte er kommendes Unheil und wollte seine Hände schon vorher in Unschuld waschen [12, S. 245]. Nach: die Hände in Unschuld waschen - ,jegliche Schuld von sich weisen, unschuldig tun". 
11. Ich bin ein blinder Passagier auf hoher See und kann nicht aussteigen [12, S. 59].

12. „Keine Spur. Fromm wie ein Osterlamm“ $[12$, S. 63].

Bei der Expansion einer phraseologischen Basis werden vor allem adjektivische (s. Beispiel 9), oder adverbiale (s. Beispiele 7, 10, 11) Attribute ergänzt sowie Komposita mit einer Phraseologismuskonstituente gebildet (s. Beispiel 12). Das Expansionselement beinhaltet in der Regel eine sich aus dem Text ergebende Information, wodurch eine dem Text dem Textinhalt verdichtende, unerwartete Kombination sprachlicher Mittel entsteht, die die Ausdruckskraft des Textes erhöht [3, S. 325].

Auch die Kombination von Expansion und Substitution kommt nicht selten vor:

13. Der Landgerichtsdirektor freute sich ungeheuer, in Dan einen Helfer des Gesetzes getroffen zu haben, wenn auch dessen Dackel noch weit vom rechten Pfade entfernt waren [12, S. 207]. Nach: vom rechten Weg(e)/Kurs abkommen - „kriminell, lasterhaft werden“.

Die Reduktion besteht darin, dass eine den Phraseologismus mit konstituierende Komponente in einem bestimmten Zusammenhang weggelassen wird [1, S. 213]. Die Reduktion setzt beim LesereinehoheSprachkompetenzvoraus. Durch die Kürze des Ausdrucks wird auf Grund des Kontrastes mit der Grundform des Phraseologismus erhöhte Expressivität erreicht:

14. Da war etwas faul im Staat $<\ldots>$ [14, S. 217].

15. Sie stellte die Teller in das obere Geschoss, immer trällernd und guter Dinge [12, S. 192]. Nach: guter Dinge sein - „munter, hoffnungsfroh, optimistisch sein; gute Laune haben“.

Kombination von mehreren Modifikationen liegt im folgenden Beispiel vor:

16. Dort ist für mich Schluss, beim besten Willen und bei aller Freundschaft [15, S. 128]. Zugrunde der Kontamination liegen zwei Phraseologismen: beim besten Willen nicht - ,ausgeschlossen“ (reduzierte Form) und in aller Freundschaft - „freundschaftlich“ (Austausch der Präposition).

Mit solchen Modifikationen wird nicht nur der expressive Effekt erzeugt, sondern sie könnender Informationsverdichtung und Textverflechtung dienen.

Fazit und Ausblick. Als stilistische und textkonstitutive Mittelsind die Phraseologismen ein fester sprachlicher Bestandteil der literarischen Texte. Die kreative Verwendung der Phraseologismen weckt Aufmerksamkeit der Leser, sie haben expressive, pragmatische, informationsverdichtende, textorganisierende und kohärenzerzeugende Funktionen. Die phraseologischen Modifikationen sind bewusst vorgenommene Veränderungen, bei denen die Identität des Phraseologismus nicht beeinträchtigt wird, sondern zum Ausdruck kommt, denn gerade die Modifikationen, die phraseologischen Einheiten als sprachliche Zeichen in den Texten zulassen, stellen eines ihrer charakterisierenden Merkmale dar und sind aufgrund der Aktivierung ihrer textbildenden Potenzen möglich.

Die Perspektiven der weiteren Forschungen liegen in der Analyse der phraseologischen Modifikationen in anderen Texttypen.

\section{Literatur:}

1. Fleischer W. Phraseologie der deutschen Gegenwartssprache: 2. durchgelesene und ergänzte Auflage. Tübingen : Max Niemeyer Verlag, 1997. $299 \mathrm{~S}$.

2. Čhernyševa I. Feste Wortkomplexe des Deutschen in Sprache und Rede. M.: Vysšaja škola, 1980. 144 S

3. Barz I. Probleme der phraseologischen Modifikation. Deutsch als Fremdsprache. 1986. Jg. 23. № 6. S. 321-336.

4. Palm Meister Ch. Phraseologie im literarischen Text am Beispiel von Morgenstern, Kafka, Brecht, Thomas Mann und Christa Wolf Phraseme und typisierte Rede / Hrsg. Nicole Fernandez Bravo. Tübingen : Stauffenburg-Verlag, 1999. S. 111-120.

5. Dobrovol'skij D. Zur Dialektik des Begriffs der textbildenden Potenzen von Phraseologismen. Zeitschrift für Phonetik, Sprachwissenschaft und Kommunikationsforschung. Bd. 33. Heft 6. Berlin : AkademieVerlag, 1980. S. 690-700.

6. Gréziano G. Forschungen zur Phraseologie. ZGL 11.2. Berlin (West)/ New York, 1983. S. 232-241.

7. Fleischer W., Helbig G., Lerchner G. Kleine Enzyklopädie - deutsche Sprache. Frankfurt am Main : Europäischer Verlag für Wissenschaften, 2001. $845 \mathrm{~S}$.

8. Wotjak B. Verbale Phraseolexeme in System und Text. Tübingen : Niemeyer, 1992. $203 \mathrm{~S}$.

9. Pociask J. Phraseologismen in deutschen und polnischen Pressetexten, dargestellt aus pragmalinguistischer Perspektive. Linguistik Online. № 4 (5). DOI: https://doi.org/10.13092/lo.74.2227.

10. Burger H. u. a. Handbuch der Phraseologie. Berlin (West) / New York : Walter de Gruyter-Verlag, 1982. 433 S.

11. Sabban A. Textbildende Potenzen von Phrasemen. Phraseologie: ein internationales Handbuch der zeitgenössischen Forschung = Phraseology: an international handbook of contemporary research / ed. by Harald Burger et al. Berlin : Walter de Gruyter, 2007. S. 237-253.

\section{Werke:}

12. Gruhl H. Liebe und Ehe auf krummen Beinen. Verlag : Deutscher Bücherbund, 1977.256 S

13. Claudius E. Menschen an unserer Seite. Stuttgart : Verlag Neuer Weg, 1984. $343 \mathrm{~S}$.

14. Mann H. Der Untertan. Leipzig : Philipp Recklam Verlag, 1967. 441 S.

15. Konsalik H. Wie ein Hauch von Zauberblüten. München : Verlag: Goldmann, 1981.415 S

Lalaian N. S. Phraseological units and text: problems of phraseological modifications

Summary. The article deals with the functioning of German phraseological units in literary texts. The main differences of idioms from free phrases (lexical-semantic integrity, idiomaticity, lexicalization, reproducibility) are determined; peculiarities of functioning of idioms in artistic texts are analyzed; questions of text-forming potential of phraseological units are considered and the most important characteristics of idioms that determine their text-forming potential are highlighted; the differences between phraseological variants, variations and phraseological modifications are explained, and approaches to the interpretation of these concepts by various linguists are explained; the main types of modifications of phraseological units are analyzed on the example of literary texts. It is proved that speech transformations are not accidental or erroneous facts, but a typical way of using idioms, which is caused by a certain communicative-pragmatic purpose and belongs to the system-defined properties of idioms. The main reasons for the formation and use of phraseological modifications are: influence on the recipient, enhancement of expressiveness and imagery of expression, compaction of information, achievement of coherence of the text, obtaining a certain wordplay effect, nomination. On the basis of examples from the works of German authors the phenomena of phraseological substitution (lexical, pragmatically conditioned or predetermined by the text of replacement of the components of the reverse), expansion (extension of the component composition of idioms), reduction (reduction of the component composition of idioms), contamination (merging in one expression of two or more idioms) and their combinations are analyzed.

Key words: phraseological units, functioning, literary text, text-forming potential, modification, substitution, expansion, reduction, contamination. 